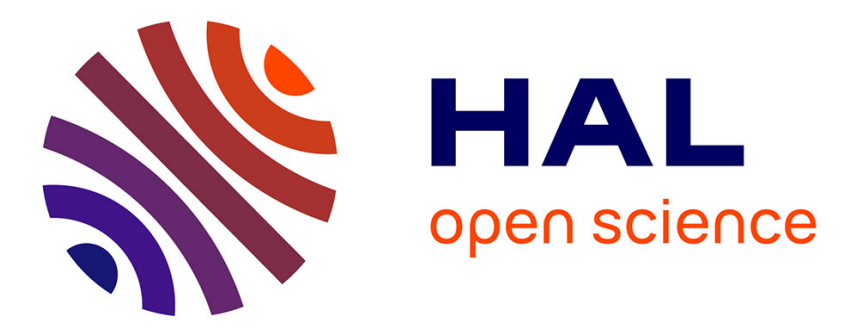

\title{
Recherche et réalisation de nouveaux alliages de niobium résistant à haute température
}

\author{
E. Alhéritière, R. Syre
}

\section{To cite this version:}

E. Alhéritière, R. Syre. Recherche et réalisation de nouveaux alliages de niobium résistant à haute température. Revue de Physique Appliquée, 1970, 5 (3), pp.477-486. 10.1051/rphysap:0197000503047700 . jpa-00243418

HAL Id: jpa-00243418

https://hal.science/jpa-00243418

Submitted on 1 Jan 1970

HAL is a multi-disciplinary open access archive for the deposit and dissemination of scientific research documents, whether they are published or not. The documents may come from teaching and research institutions in France or abroad, or from public or private research centers.
L'archive ouverte pluridisciplinaire $\mathbf{H A L}$, est destinée au dépôt et à la diffusion de documents scientifiques de niveau recherche, publiés ou non, émanant des établissements d'enseignement et de recherche français ou étrangers, des laboratoires publics ou privés. 


\title{
RECHERCHE ET RÉALISATION DE NOUVEAUX ALLIAGES DE NIOBIUM RÉSISTANT A HAUTE TEMPÉRATURE*
}

\author{
E. ALHÉRITIÈRE et R. SYRE
}

Division des Etudes et Fabrications de Métaux Spéciaux du Département des Recherches et Produits Avancés de la Société Trefimétaux GP, Argenteuil, Val d'Oise

\begin{abstract}
Résumé. - Cette étude, financée par la D. G. R. S. T., avait pour but de développer de nouveaux alliages de niobium pour l'utilisation à haute température. Les caractéristiques recherchées étaient : une résistance au fluage élevée, une bonne malléabilité et une densité raisonnable.

Parmi les compositions retenues, deux présentent des compromis intéressants :

- le WD $15(\mathrm{Nb} 9 \mathrm{~W} 6 \mathrm{Mo} 4 \mathrm{Hf} 0,2 \mathrm{C})$. Cet alliage, traité 2 hà $2000^{\circ} \mathrm{C}$ et trempé à l'huile a,

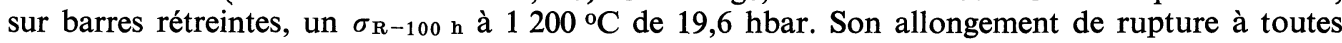
températures, dans cet état de traitement, est au moins égal à $10 \%$;

- le W6 B (Nb 6 W 2 Zr 2 Hf 0,2 C, 0,2 B). Cet alliage, dont la malléabilité est excellente, a un $\sigma_{\mathrm{R}^{-}} 100 \mathrm{~h}$ à $1200^{\circ} \mathrm{C}$ de 14,6 hbar sur tôles laminées, traitées $2 \mathrm{~h}$ à $2000^{\circ} \mathrm{C}$ et trempées à l'huile.

Abstract. - The present study, sponsored by the D. G. R. S. T., was designed to develop niobium base alloys suitable for application at high temperatures. Properties required were : high creep strength, good fabricability and moderate density.

Two alloy formulations are attractive :

- WD 15 ( Nb 9 W 6 Mo 4 Hf $0.2 \mathrm{C}$ ). This alloy, heat treated 2 hours at $2000{ }^{\circ} \mathrm{C}$ and oil quenched, has a one hundred hours rupture strength of 19.6 hbar. Rupture elongation is over ten per cent at any temperature.

- W 6 B (Nb 6 W 2 Zr 2 Hf 0.2 C, 0.2 B). This alloy, which has an excellent workability, has a one hundred hours rupture strength of $14.5 \mathrm{hbar}$, on rolled sheets treated 2 hours at $2000{ }^{\circ} \mathrm{C}$ and oil quenched.
\end{abstract}

1. Introduction. - L'utilisation des alliages de niobium à hautes températures nécessite la mise au point de matériaux ayant à la fois une bonne tenue au fluage, une densité raisonnable et une malléabilité convenable.

Un contrat passé avec la DGRST nous a permis de rechercher de nouvelles nuances répondant mieux à ces trois critères que celles existant actuellement.

La recherche a été conduite de la façon suivante :

1) Etude préliminaire d'une trentaine d'alliages expérimentaux, sur boutons.

2) Choix de quatre compositions et fusion d'un lingot industriel dans chaque nuance.

3) Détermination des propriétés de ces quatre alliages.

2. Etude préliminaire de trente alliages. 2.1 CHOIX DES ÉLÉMENTS D'ADDITIONS. - Les deux façons habituelles de durcir un alliage ont été utilisées :

* Communication présentée aux Journées du Niobium, organisées par la D. R. M. E. et la D. G. R. S. T. à Paris, les 20 et 21 octobre 1969. l'introduction, d'une part, d'éléments durcisseurs de la solution solide, d'autre part, d'éléments pouvant permettre d'obtenir des précipitations stables.

Notre but étant de durcir le niobium dans son comportement à haute température, nous avons choisi principalement :

- Des éléments qui diffusent peu et dont l'effet de distorsion de la matrice reste encore marqué à haute température.

Le tungstène et le molybdène ont été retenus. Pour limiter la densité des alliages et ne pas compromettre leur malléabilité, nous avons limité à $10 \%$ la teneur en chacun de ces éléments et à $15 \%$ la somme (tungstène + molybdène) dans le même alliage.

- Des dispersions les plus stables possibles aux températures d'utilisation envisagées, tels les carbures et les borures de zirconium et de hafnium. Pour des raisons de malléabilité à chaud et de résistance à l'oxydation, nous avions décidé d'introduire du titane. Mais cet élément diminuant la résistance au fluage du niobium, il a été associé au vanadium, qui a un effet durcissant. Les difficultés rencontrées avec ces éléments seront évoquées plus loin. 
TABleau I

Composition nominale des 30 alliages de niobium étudiés

\begin{tabular}{|c|c|c|c|c|c|c|c|c|c|c|}
\hline $\begin{array}{c}\text { Alliage } \\
n^{0}\end{array}$ & $\begin{array}{l}\mathrm{W} \\
\%\end{array}$ & $\begin{array}{c}\text { Mo } \\
\%\end{array}$ & $\begin{array}{l}\mathrm{Hf} \\
\%\end{array}$ & $\begin{array}{c}\mathrm{Zr} \\
\%\end{array}$ & $\begin{array}{l}\mathrm{Ti} \\
\%\end{array}$ & $\begin{array}{l}\mathrm{V} \\
\%\end{array}$ & $\begin{array}{r}\mathrm{Be} \\
\%\end{array}$ & $\begin{array}{c}\mathrm{Al} \\
\%\end{array}$ & $\begin{array}{c}\mathrm{C} \\
\mathrm{ppm}\end{array}$ & $\underset{\mathrm{ppm}}{\mathbf{B}}$ \\
\hline 1 & 10 & 5 & 3 & 3 & 3 & 5 & - & - & 4000 & - \\
\hline 2 & 8 & 5 & 3 & 3 & 3 & 5 & - & - & 4000 & - \\
\hline 3 & 6 & 6 & 2 & 3 & 3 & 5 & - & - & 4000 & - \\
\hline 4 & 3 & 3 & 10 & 3 & 3 & 3 & - & - & 5000 & - \\
\hline 5 & 8 & 5 & 3 & 3 & 5 & 5 & - & - & 4000 & - \\
\hline 6 & 5 & 5 & 5 & 3 & 10 & 5 & - & - & 3500 & 500 \\
\hline 7 & 10 & 5 & 2 & 5 & 10 & 3 & - & - & 4000 & - \\
\hline 8 & 10 & 3 & 2 & 5 & 10 & 3 & - & - & 4000 & - \\
\hline 9 & 3 & 3 & 5 & 3 & 10 & 3 & - & - & 4000 & - \\
\hline 10 & 10 & - & 2 & 3 & 3 & - & - & - & 3000 & 1000 \\
\hline 11 & 10 & - & 2 & 5 & 3 & - & - & - & 5000 & 1000 \\
\hline 12 & 5 & - & 2 & 3 & 3 & - & - & - & 3000 & 1000 \\
\hline 13 & 5 & - & 2 & 5 & 3 & - & - & - & 5000 & 1500 \\
\hline 14 & 10 & 2 & 3 & 3 & 5 & - & - & - & 4000 & 500 \\
\hline 15 & 10 & 2 & 3 & 3 & 5 & - & - & - & 5000 & - \\
\hline 16 & 10 & - & 3 & 3 & 5 & - & - & - & 3000 & 1500 \\
\hline 17 & 10 & - & 3 & 5 & 5 & - & - & - & 5000 & 1500 \\
\hline 18 & 5 & - & 3 & 3 & 5 & - & - & - & 3000 & 1500 \\
\hline 19 & 5 & - & 3 & 5 & 5 & - & - & - & 5000 & 1500 \\
\hline 20 & 10 & 2 & 3 & - & 5 & 5 & - & - & 1500 & 500 \\
\hline 21 & 10 & 2 & 3 & - & 5 & 5 & - & - & 1500 & - \\
\hline 22 & 8 & 5 & 5 & - & 5 & 3 & - & - & 2500 & 500 \\
\hline 23 & 8 & 5 & 5 & - & 5 & 3 & - & - & 2500 & - \\
\hline 24 & - & 10 & - & 2 & 3 & 3 & - & 2 & 2000 & - \\
\hline 25 & - & 10 & 2 & 2 & 3 & 3 & - & 2 & 3000 & - \\
\hline 26 & - & 10 & 2 & 2 & 3 & 3 & 0,20 & 2 & 3000 & - \\
\hline 27 & - & 10 & - & 2 & 3 & 3 & - & 2 & 4000 & - \\
\hline 28 & - & 10 & 2 & 2 & 3 & 3 & - & 2 & 5000 & - \\
\hline 29 & - & 10 & 2 & 2 & 3 & 3 & 0,20 & 2 & 5000 & - \\
\hline 30 & - & 10 & 2 & 2 & 3 & 3 & 0,20 & 2 & 5000 & - \\
\hline
\end{tabular}

Enfin nous avons tenté d'introduire de l'aluminium et du béryllium en petites quantités.

Les compositions retenues sont indiquées dans le tableau I.

2.2 Elaboration. - Pour chaque composition, nous avons élaboré quatre boutons de $100 \mathrm{~g}$ chacun, par double fusion au four à bombardement électronique. A ce stade, des difficultés d'élaboration sont apparues, le titane, le vanadium, l'aluminium et le béryllium ayant tendance à s'évaporer, malgré toutes les précautions prises.

2.3 ANALYSE. - Les dosages classiques par voie chimique ne conviennent pas à l'analyse de tels alliages, il a donc fallu mettre au point une méthode mieux adaptée : la fluorescence $\mathrm{X}$ nous a apporté une solution nouvelle, répondant tout à fait à ce que l'on cherchait ; l'analyse est à la fois rapide et précise. A l'aide d'un étalonnage assez complet, nous pouvons maintenant faire l'analyse directe non destructive sur un disque de $30 \mathrm{~mm}$ de diamètre, simplement poli. Cette analyse est limitée aux éléments plus lourds que le magnésium, l'absorption de la matrice ne permettant pas de doser les éléments plus légers.

Ainsi, le carbone, l'oxygène et l'azote ont été dosés par voie chimique. Mais pour ces éléments, l'analyse chimique ne présente pas de difficultés particulières. Seul le bore n'a pu être dosé de façon satisfaisante. La méthode employée (spectrométrie optique) donne seulement une indication qualitative. Si les alliages au bore devaient être développés, il faudrait absolument mettre au point une méthode convenable, peutêtre par absorption atomique.

L'ensemble des teneurs analysées figure dans le tableau II.

2.4 Difficultés RencontréES. - L'examen du tableau II montre que les pertes en titane et vanadium ont été très importantes. Ces éléments ont des tensions de vapeurs telles qu'ils s'évaporent en grande partie. 
TABLEAU II

Analyse des 30 alliages de niobium initiaux

\begin{tabular}{|c|c|c|c|c|c|c|c|c|}
\hline $\begin{array}{c}\text { Alliage } \\
n^{0}\end{array}$ & $\begin{array}{l}\mathrm{Ti}^{*} \\
\%\end{array}$ & $\begin{array}{l}V^{*} \\
\%\end{array}$ & $\begin{array}{c}\mathrm{Zr}^{*} \\
\%\end{array}$ & $\begin{array}{c}\mathrm{Hf}^{*} \\
\%\end{array}$ & $\begin{array}{c}\mathrm{W}^{*} \\
\%\end{array}$ & $\begin{array}{c}\text { Mo* } \\
\%\end{array}$ & $\begin{array}{l}\mathrm{C}^{* *} \\
\mathrm{ppm}\end{array}$ & $\begin{array}{l}B^{* *} \\
\text { ppm }\end{array}$ \\
\hline 1 & 1,4 & 2,4 & 2,7 & 3,15 & 10,8 & 4,45 & 3500 & - \\
\hline 2 & - & - & 2,2 & 3,0 & 9,2 & 4,75 & 3400 & - \\
\hline 3 & - & 0,15 & 2,55 & 2,1 & 6,8 & 5,9 & 3300 & - \\
\hline 4 & 0,75 & 0,4 & 2,8 & 10,2 & 3,0 & 2,9 & 4200 & - \\
\hline 5 & 0,25 & 0,35 & 2,7 & 3,15 & 9,5 & 5,0 & 3200 & - \\
\hline 6 & 4,0 & 2,2 & 2,30 & 5,4 & 5,9 & 4,65 & 3100 & 1600 \\
\hline 7 & - & - & 3,55 & 2,4 & 13,5 & 7,6 & 3600 & - \\
\hline 8 & 1,0 & 0,4 & 4,60 & 2,5 & 11,9 & 3,2 & 3600 & - \\
\hline 9 & 3,25 & 1,2 & 5,25 & 5,9 & 3,45 & 3,2 & 3900 & - \\
\hline 10 & - & - & 2,4 & 1,9 & 10,7 & - & 3000 & 1400 \\
\hline 11 & - & - & 3,7 & 2,0 & 11,0 & - & 4200 & 1200 \\
\hline 12 & - & - & 2,2 & 1,85 & 5,4 & - & 3000 & 1100 \\
\hline 13 & - & - & 3,4 & 2,0 & 5,6 & - & 4400 & 1300 \\
\hline 14 & 0,3 & - & 1,55 & 2,5 & 11,6 & 1,5 & 3200 & 1500 \\
\hline 15 & 0,25 & - & 1,85 & 2,7 & 10,9 & 1,6 & 2900 & - \\
\hline 16 & 0,15 & - & 2,5 & 2,9 & 10,9 & - & 3600 & 3600 \\
\hline 17 & - & - & - & - & - & - & 4100 & 1500 \\
\hline 18 & 0,15 & - & 2,7 & 3,0 & 6,2 & - & 2900 & 1500 \\
\hline 19 & - & - & 4,2 & 3,2 & 5,3 & - & 4200 & 1400 \\
\hline 20 & 0,8 & 1,2 & - & 2,7 & 11,0 & 1,8 & 1600 & 1800 \\
\hline 21 & 0,1 & 0,1 & - & 2,8 & 11,7 & 1,85 & 1500 & - \\
\hline 22 & 0,4 & 0,45 & - & 4,85 & 9,9 & 4,8 & 2100 & 1300 \\
\hline 23 & - & - & - & 4,0 & 9,7 & 4,2 & 2500 & - \\
\hline 24 & - & - & 2,0 & - & - & 9,5 & 1800 & - \\
\hline 25 & 0,3 & 0,35 & 2,3 & 2,0 & - & 9,7 & 3100 & - \\
\hline 26 & 0,3 & 0,3 & 2,4 & 1,95 & - & 9,7 & 3000 & - \\
\hline 27 & - & 0,25 & 2,2 & - & - & 9,7 & 3800 & - \\
\hline 28 & 0,9 & 0,75 & 2,9 & 2,02 & - & 9,8 & 4000 & - \\
\hline 29 & 0,25 & 0,3 & 2,3 & 1,9 & - & 9,8 & 4500 & - \\
\hline 30 & 0,15 & 0,2 & 2,3 & 1,9 & - & 9,8 & 4400 & 2700 \\
\hline
\end{tabular}

* Analyse par fluorescence X

** Analyse par voie chimique

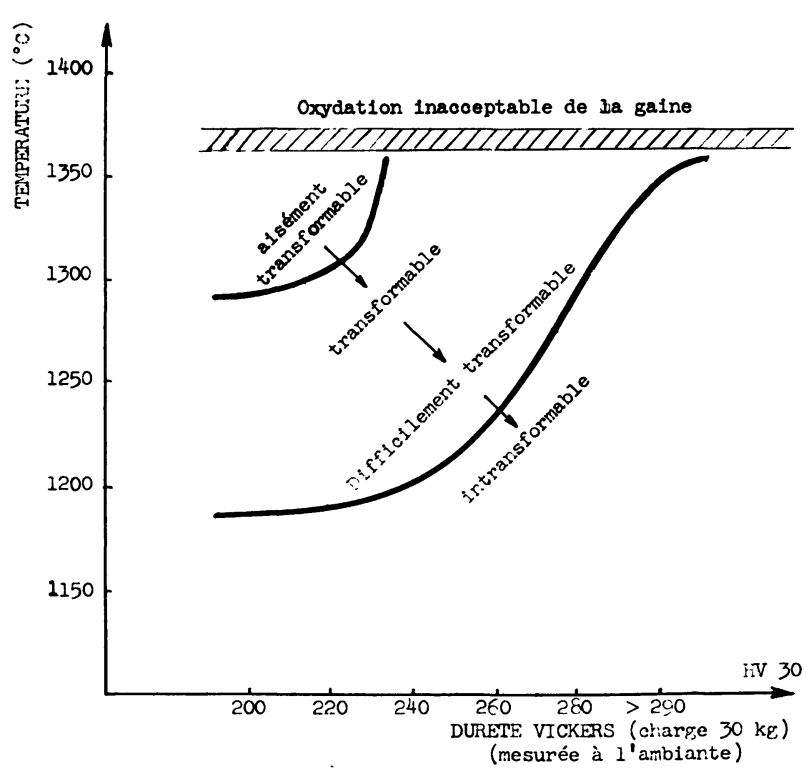

FIG. 1. - Conditions de forgeage.
Malgré cela, nous avons poursuivi la transformation de ces alliages, pour étudier l'influence des autres éléments d'additions sur les caractéristiques à chaud des alliages de niobium.

2.5 Transformation. - Le comportement au forgeage des trente alliages est résumé sur la figure 1 . Les boutons ont été ensuite laminés à chaud de 4 à $1,5 \mathrm{~mm}$ d'épaisseur, puis à tiède de 1,5 à $1 \mathrm{~mm}$ d'épaisseur.

2.6 CARACTÉRISATION. - Sur les tôles obtenues, nous avons découpé des plaquettes pour essai de soudage, pliage et oxydation, et des éprouvettes de fluage-rupture.

2.6.1 Essai de soudage et pliage du cordon. - Tous les alliages sont soudables à l'argon arc. Les résultats des essais de pliage du cordon de soudure, mesurés par l'angle de pliage à la rupture, sont indiqués dans le tableau III. 


\section{TABleaU III}

Essais de pliage sur tôles soudées

(Angle de pliage à la rupture. Poinçon de rayon $R=6, e=6 \mathrm{~mm}$; vitesse de pénétration : $2 \mathrm{~mm} / \mathrm{mn}$ ).

$\begin{array}{cc}\begin{array}{c}\text { Pliage } \\ \mathrm{n}^{\mathrm{o}}\end{array} & \begin{array}{c}\text { perpendiculairement } \\ \text { au cordon de soudure }\end{array} \\ \frac{-}{4} & -80 \\ 10 & >90 \\ 11 & >90 \\ 12 & >90 \\ 13 & >90 \\ 14 & 50 \\ 15 & 65 \\ 17 & 40 \\ 18 & >90\end{array}$

$\begin{array}{cc}\begin{array}{cc}\text { Alliage } \\ \mathbf{n}^{\circ}\end{array} & \begin{array}{c}\text { Pliage } \\ \text { perpendiculairement } \\ \text { au cordon de soudure }\end{array} \\ - & - \\ 21 & >90 \\ 22 & 10 \\ 23 & 40 \\ 24 & >90 \\ 25 & 10 \\ 26 & 30 \\ 27 & 45\end{array}$

2.6.2 Essais d'oxydation. - Ils ont été faits à titre indicatif : nous avons enregistré, pendant deux heures, le gain de poids de plaquettes $10 \times 10 \times 1 \mathrm{~mm}$, maintenues à $1000^{\circ} \mathrm{C}$ et $1200^{\circ} \mathrm{C}$ en air calme (tableau IV).

\section{TABLEAU IV}

Vitesse d'oxydation à 1000 et $1200{ }^{\circ} \mathrm{C}$ (exprimée en $\mathrm{mg} \mathrm{cm}^{-2} \mathrm{~h}^{-1}$; essai de $2 \mathrm{~h}$ en air calme)

\begin{tabular}{|c|c|c|c|c|c|}
\hline Alliage & & & Alliage & & \\
\hline $\mathrm{n}^{\mathrm{o}}$ & $1000^{\circ} \mathrm{C}$ & $1200^{\circ} \mathrm{C}$ & $n^{0}$ & $1000{ }^{\circ} \mathrm{C}$ & $1200{ }^{\circ} \mathrm{C}$ \\
\hline- & 一 & - & - & - & - \\
\hline 1 & 14 & 22 & 16 & 27 & 29 \\
\hline 2 & 11 & 24 & 17 & 21 & 27 \\
\hline 3 & 7 & 22 & 18 & 43 & 37 \\
\hline 4 & 11 & 28 & 19 & 25 & 34 \\
\hline 5 & 8 & 32 & 20 & 13 & 30 \\
\hline 6 & 10 & 36 & 21 & 9 & 20 \\
\hline 7 & 14 & 30 & 22 & 9 & 20 \\
\hline 8 & 10 & 21 & 23 & 7 & 19 \\
\hline 9 & 8 & 21 & 24 & 7 & 21 \\
\hline 10 & 32 & 29 & 25 & 18 & 28 \\
\hline 11 & 21 & 26 & 26 & 14 & 25 \\
\hline 12 & 40 & 32 & 28 & 16 & 25 \\
\hline 13 & 44 & 31 & 29 & 18 & 28 \\
\hline 14 & 13 & 20 & 30 & 20 & 31 \\
\hline 15 & 9 & 24 & & & \\
\hline
\end{tabular}

La résistance à l'oxydation des alliages est de 3 à 5 fois plus faible que celle du niobium non allié à $1200^{\circ} \mathrm{C}$ et jusqu'à 9 fois plus faible à $1000^{\circ} \mathrm{C}$. Cependant, les vitesses d'oxydation sont quand même cent fois plus fortes que celles qu'on peut admettre sur une aube de turbine.

2.6.3 Essais de fluage-rupture. - Les éprouvettes ont été traitées avant essai $1 \mathrm{~h}$ à $1800^{\circ} \mathrm{C}$ (refroidissement au four). Nous avons déterminé la contrainte donnant une durée de vie de $100 \mathrm{~h}$ à $1200^{\circ} \mathrm{C}$ (tableau V).

\section{TABleaU $\mathrm{V}$}

Tenue au fluage des alliages

de niobium à $1200^{\circ} \mathrm{C}$

$\begin{array}{cccc}\begin{array}{c}\text { Alliage } \\ \mathrm{n}^{\mathbf{0}}\end{array} & \begin{array}{c}1200^{\circ} \mathrm{C} \\ \frac{\sigma_{\mathrm{R}}-100 \mathrm{~h}}{\text { (hbar) }}\end{array} & \begin{array}{c}\text { Alliage } \\ \mathrm{n}^{\mathbf{0}}\end{array} & \begin{array}{c}1200{ }^{\circ} \mathrm{C} \\ \sigma_{\mathrm{R}-100 \mathrm{~h}} \text { (hbar) }\end{array} \\ 3 & 15,5 & \overline{18} & \overline{7,8} \\ 4 & 15,9 & 19 & 9,8 \\ 9 & 7,4 & 20 & 15,2 \\ 10 & 8,3 & 21 & 14,8 \\ 11 & 14,7 & 22 & 15,2 \\ 12 & 11,3 & 23 & 17,7 \\ 13 & 11,3 & 24 & 13,5 \\ 14 & 10,8 & 25 & 14,2 \\ 15 & 15,2 & 26 & 13,7 \\ 16 & 15,5 & 27 & 15,7 \\ 17 & 12,5 & 28 & 14,2 \\ & 11,4 & 29 & 15,2\end{array}$

Sur vingt alliages, pour lesquels la détermination de $\sigma_{\mathrm{R}-100 \mathrm{~h}}$ était particulièrement sûre, nous avons analysé de façon précise l'influence des éléments d'additions sur la tenue au fluage à $1200^{\circ} \mathrm{C}$ (caractérisé par $\sigma_{\mathrm{R}-100 \mathrm{~h}}$ ). Les diverses corrélations faites on permis de mettre en lumière les points suivants :

- Le molybdène et le tungstène augmentent sensiblement $\sigma_{\mathrm{R}-100 \mathrm{~h}}^{1200 \mathrm{C}}$

Leur influence est comparable, l'addition de molybdène étant légèrement plus efficace que celle du tungstène (à même teneur pondérale).

- On a intérêt à baisser le rapport atomique $(\mathrm{Hf}+\mathrm{Zr} / \mathrm{C})$ au-dessous de 1,5 et à le maintenir de préférence entre 1 et 1,2 .

3. Choix de quatre alliages. - 3.1 Chaque alliage a été noté selon deux critères : résistance au fluage et malléabilité, en employant, pour chacun d'eux, cinq qualificatifs : négligeable, médiocre, passable, bon et excellent (tableau VI).

Les alliages présentant les meilleurs compromis entre ces deux critères sont : le $n^{0} 12$ (très malléable) les $\mathrm{n}^{0} 15,23$ et 27 (très résistants au fluage).

3.2 Fusion. - Dans chacune des quatre compositions retenues, un lingot de trente kilogrammes a été élaboré au four à bombardement d'électrons, en lingotière de $80 \mathrm{~mm}$ de diamètre. Pour améliorer l'homogénéisation, les précautions suivantes ont été prises :

- utilisation d'alliages-mères ;

- double fusion, avec mise en copeaux et recompression après la première fusion.

Les analyses finales, faites sur deux disques pour chaque alliage, sont indiquées dans le tableau VII. 
TABLEAU VI

Qualification des alliages de niobium

\begin{tabular}{|c|c|c|c|c|c|c|c|c|}
\hline \multirow{3}{*}{$\begin{array}{c}\text { Alliage } \\
n^{0}\end{array}$} & \multicolumn{6}{|c|}{ Analyse par fluorescence $\mathrm{X}$} & \multicolumn{2}{|c|}{ Critères retenus } \\
\hline & Ti. & V & $\mathrm{Zr}$ & W & $\mathrm{Hf}$ & Mo & Résistance & Malléabilité \\
\hline & $\%$ & $\%$ & $\%$ & $\%$ & $\%$ & $\%$ & au fluage & \\
\hline 1 & 1,4 & 2,4 & 2,7 & 10,8 & 2,15 & 4,45 & - & négligeable \\
\hline 2 & - & - & 2,2 & 9,2 & 3,0 & 4,75 & bonne & bonne \\
\hline 3 & - & 0,15 & 2,55 & 6,8 & 2,1 & 5,9 & excellente & passable \\
\hline 4 & 0,75 & 0,4 & 2,8 & 3,0 & 10,2 & 2,9 & négligeable & médiocre \\
\hline 5 & 0,25 & 0,35 & 2,7 & 9,5 & 3,15 & 5,0 & - & médiocre \\
\hline 6 & 4,0 & 2,2 & 2,3 & 5,9 & 5,4 & 4,65 & - & négligeable \\
\hline 7 & - & - & 3,55 & 13,5 & 2,4 & 7,6 & - & négligeable \\
\hline 8 & 1,0 & 0,4 & 4,6 & 11,9 & 2,5 & 3,2 & - & négligable \\
\hline 9 & 3,25 & 1,2 & 5,25 & 3,45 & 5,9 & 3,2 & négligeable & passable \\
\hline 10 & - & - & 2,4 & 10,7 & 1,9 & - & bonne & bonne \\
\hline 11 & - & - & 3,7 & 11,0 & 2,0 & - & passable & bonne \\
\hline $12 *$ & - & - & 2,2 & 5,4 & 1,85 & - & passable & excellente \\
\hline 13 & - & - & 3,4 & 5,6 & 2,0 & - & passable & excellente \\
\hline 14 & 0,3 & - & 1,55 & 11,6 & 2,5 & 1,5 & médiocre & passable \\
\hline $15^{*}$ & 0,25 & - & 1,85 & 10,9 & 2,7 & 1,6 & excellente & bonne \\
\hline 16 & 0,15 & - & 2,5 & 10,9 & 2,9 & - & passable & bonne \\
\hline 17 & - & - & - & - & - & - & passable & passable \\
\hline 18 & 0,15 & - & 2,7 & 6,2 & 3,0 & - & négligeable & excellente \\
\hline 19 & - & - & 4,2 & 5,3 & 3,2 & - & médiocre & excellente \\
\hline 20 & 0,8 & 1,2 & - & 11,0 & 2,7 & 1,8 & bonne & passable \\
\hline 21 & 0,1 & 0,1 & - & 11,7 & 2,8 & 1,85 & bonne & bonne \\
\hline 22 & 0,4 & 0,45 & - & 9,9 & 4,85 & 4,8 & bonne & bonne \\
\hline $23 *$ & - & - & - & 9,7 & 4,0 & 4,2 & excellente & bonne \\
\hline 24 & - & - & 2,0 & - & 9,5 & 9,5 & passable & bonne \\
\hline 25 & 0,3 & 0,35 & 2,3 & - & 2,0 & 9,7 & bonne & bonne \\
\hline 26 & 0,3 & 0,3 & 2,4 & - & 1,95 & 9,7 & bonne & bonne \\
\hline $27^{*}$ & - & 0,25 & 2,2 & - & - & 9,7 & excellente & passable \\
\hline 28 & 0,9 & 0,75 & 2,9 & - & 2,02 & 9,8 & bonne & médiocre \\
\hline 29 & 0,25 & 0,3 & 2,3 & - & 1,9 & 9,8 & bonne & bonne \\
\hline 30 & 0,15 & 0,2 & 2,3 & - & 1,9 & 9,8 & - & négligeable \\
\hline
\end{tabular}

* Alliages retenus.

TABLEAU VII

Analyse des quatre alliages de niobium retenus

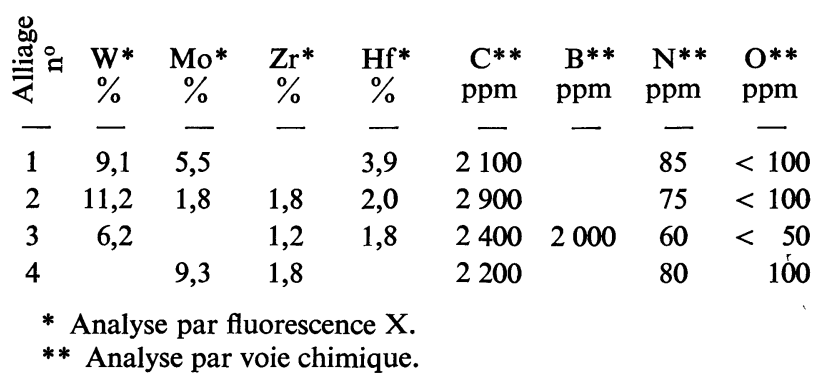

Les pertes à la fusion ont été très faibles et les lingots obtenus sont tout à fait sains, sauf l'alliage $\mathrm{n}^{0} 3$ qui présente quelques défauts.

Cet alliage, on le verra par la suite, est très malléable et cette mauvaise santé initiale n'a pas nui à la transformation. Cependant, son développement industriel nécessiterait une étude complémentaire sur ce point (ainsi que sur le dosage du bore, comme on l'a souligné précédemment).

3.3 Transformation. - Chaque lingot a été ensuite transformé, partie par forgeage et laminage, partie par filage et rétreint.

Les traits principaux du comportement des quatre alliages au cours de la transformation sont les suivants :

- l'adoucissement initial le plus efficace s'obtient par un traitement de 2 heures à $2000^{\circ} \mathrm{C}$, suivi d'un refroidissement lent $\left(150^{\circ} \mathrm{C} / \mathrm{h}\right)$ (tableau VIII) ;

- la première transformation (forgeage et filage) doit se faire à haute température : à plus de $1300^{\circ} \mathrm{C}$ pour les alliages nos 1,2 et 4 , à plus de $1200^{\circ} \mathrm{C}$ pour l'alliage $n^{\circ} 3$; 
- la seconde transformation, laminage et rétreint, peut se faire entre 1000 et $1200^{\circ} \mathrm{C}$, voire même à l'ambiante pour l'alliage $n^{\circ} 3$;

- la capacité d'écrouissage à froid est de $90 \%$ sur l'alliage $n^{0} 3$ à partir de $10 \mathrm{~mm}$, et de 75 à $80 \%$ sur les trois autres alliages à partir de $4 \mathrm{~mm}$. Sur les barres rétreintes, la capacité d'écrouissage de l'alliage no 3 est encore de $90 \%$.

En revanche, les alliages nos 1,2 et 4 ne peuvent subir par rétreint qu'un léger écrouissage à froid $(20 \%)$.

En respectant ces conditions, les quatre alliages sont réellement faciles à transformer. Les tôles obtenues sont saines et exemptes de criques.

\section{TABLEAU VIII}

Traitement d'adoucissement avant transformation (Larget de $10 \mathrm{~mm}$ d'épaisseur)

\begin{tabular}{|c|c|c|c|c|}
\hline \multirow[b]{2}{*}{ 怘。 } & \multicolumn{4}{|c|}{ Dureté HV 30} \\
\hline & $\begin{array}{c}\text { Avant } \\
\text { traite- } \\
\text { ment }\end{array}$ & $1150^{2 \mathrm{~h} /} \mathrm{C} / \mathrm{RF}^{*}$ & $1450^{\circ \mathrm{h} /} / \mathrm{RF}^{*}$ & $2000^{2 \mathrm{~h} /} \mathrm{C} / \mathrm{RF}^{*}$ \\
\hline 一 & 一 & - & - & - \\
\hline 1 & 238 & 228 & 213 & 183 \\
\hline 2 & 238 & 212 & 202 & 197 \\
\hline & 170 & 150 & 143 & 143 \\
\hline 4 & 223 & 200 & 190 & 185 \\
\hline
\end{tabular}

4. Caractérisation. - Nous avons étudié, sur chaque alliage, la température de recristallisation, l'influence de divers facteurs (traitements, écrouissage) sur la tenue au fluage et déterminé plus complètement, dans le meilleur état, les caractéristiques mécaniques à froid et à chaud de ces alliages.

4.1 Température DE Recristallisation. - Elle a été mesurée sur des tôles de $2,5 \mathrm{~mm}$ d'épaisseur, recristallisées $2 \mathrm{~h}$ à $1450{ }^{\circ} \mathrm{C}$ puis écrouies à froid de $10,20,40$ et $60 \%$. Pour chaque taux de réduction, nous avons fait des recuits de $1 \mathrm{~h}$ à $1000,1200,1300$, $1400,1500,1600,1800$ et $2000^{\circ} \mathrm{C}$. La recristallisation a été suivie à l'aide de diagrammes de Laüe.

Nous avons retrouvé évidemment quelques-unes des lois classiques de la recristallisation: elle a lieu à une température d'autant plus élevée que l'écrouissage initial est plus faible ; à très haute température, le grain reste d'autant plus fin que l'écrouissage initial est plus fort. Mais deux faits marquants caractérisent plus particulièrement ces alliages :

- le domaine de recristallisation, après une déformation initiale de $60 \%$, est assez étendu : de $1200{ }^{\circ} \mathrm{C}$ à $1500^{\circ} \mathrm{C}$ pour les alliages $\mathrm{n}^{\mathrm{os}} 1,2$ et 4 , de 1100 à $1400^{\circ} \mathrm{C}$ pour l'alliage $\mathrm{n}^{\circ} 3$;

- le grain reste très fin jusqu'à $2000^{\circ} \mathrm{C}$ pour les alliages $\mathrm{n}^{\mathrm{s}} 1$ et 2 (10 à 20 microns). Il grossit un peu à partir de $1800^{\circ} \mathrm{C}$ pour les alliages $n^{\circ} 3$ et 4 .

La dureté à froid évolue sensiblement de la même façon sur les quatre alliages, après recuit à diverses températures (Fig. 2): le durcissement initial par écrouissage n'est pas effacé par des recuits jusqu'à $1100^{\circ} \mathrm{C}\left(1000^{\circ} \mathrm{C}\right.$ pour l'alliage $\left.n^{\circ} 3\right)$. Au-delà, il se produit une restauration et la dureté marque un palier jusque vers $1500^{\circ} \mathrm{C}\left(1400^{\circ} \mathrm{C}\right.$ pour l'alliage $\left.\mathrm{n}^{\circ} 3\right)$. Ensuite la dureté remonte par suite de modifications dans les phases dispersées.

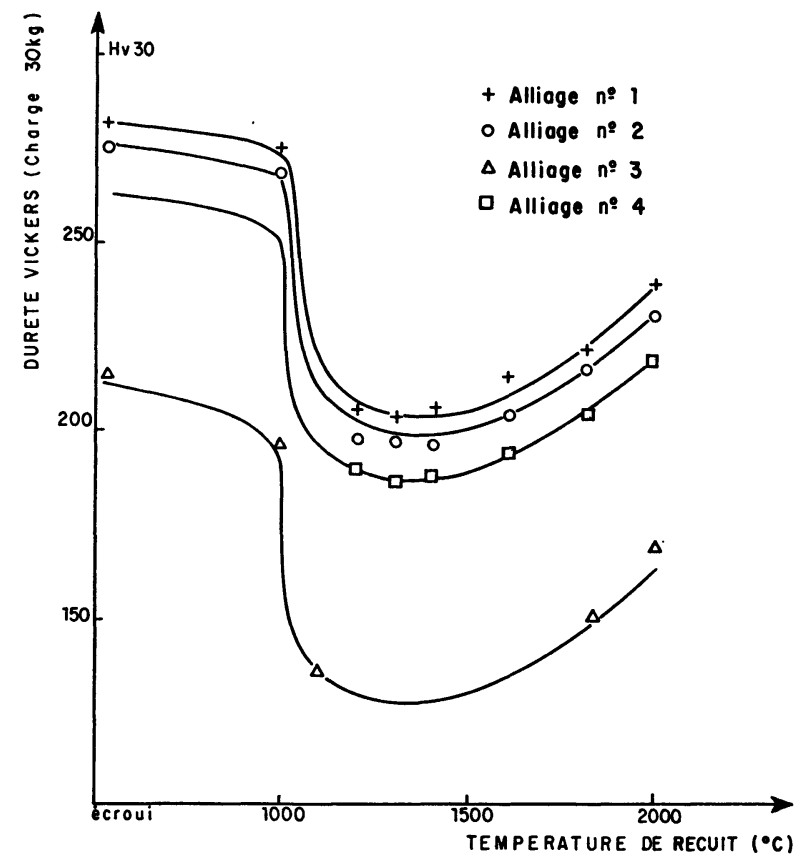

Fig. 2. - Influence du traitement thermique sur la dureté à l'ambiante. Etat initial : écroui de $60 \%$. Traitements de $1 \mathrm{~h}$ sous vide.

4.2 INFLUENCE DES TRAITEMENTS THERMO-MÉCANIQUES SUR LA TENUE A CHAUD. - L'influence des traitements thermiques et de l'écrouissage sur la tenue à chaud des quatre alliages a été étudiée par des mesures de dureté à chaud et de fluage-rupture. Les principaux résultats sont les suivants :

- le traitement qui donne la plus forte résistance au fluage à $1200^{\circ} \mathrm{C}$ est celui de $2 \mathrm{~h}$ à $2000^{\circ} \mathrm{C}$ suivi d'une trempe à l'huile ;

- le refroidissement consécutif au traitement de mise en solution à $2000^{\circ} \mathrm{C}$ est primordial : plus il est lent et plus faible est la tenue au fluage ; au contraire, plus il est rapide et meilleure est la résistance au fluage. La figure 3 illustre bien cette importance de la vitesse de refroidissement. Elle montre aussi qu'à partir d'une certaine vitesse (trempe au four) la tenue au fluage atteint un niveau qu'elle ne dépasse guère pour des vitesses de refroidissement plus élevées.

Ceci est important pour le développement industriel de ces alliages: un simple refroidissement au four (RF) (avec cependant arrêt brutal du chauffage) donne une tenue voisine de la trempe à l'huile (TH);

- des revenus de $4 \mathrm{~h}$ à $1250,1300,1350$ et $1400^{\circ} \mathrm{C}$ ont tendance à faire baisser légèrement la tenue au fluage des quatre alliages (tableau IX). 


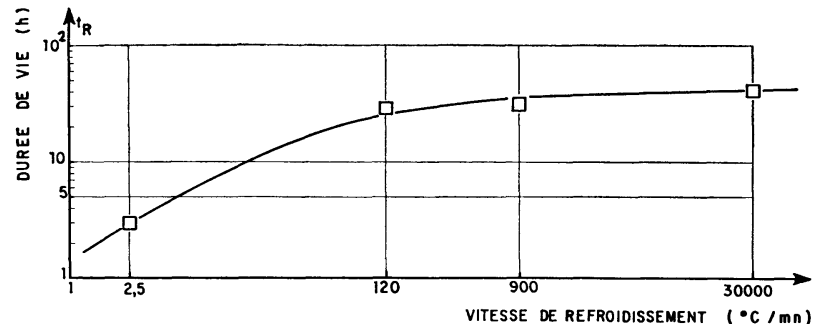

Fig. 3. - Influence de la vitesse de refroidissement sur les caractéristiques de rupture à temps (alliage $\mathrm{n}^{\circ} 4$. Charge $17,65 \mathrm{hbar}$ à $\left.1200^{\circ} \mathrm{C}\right)$.

\section{TABLEAU IX}

Influence d'un revenu sur la tenue à chaud des alliages de niobium $n^{\text {os }} 1$ et 3 (tôle d'1 mm d'épaisseur)

$\begin{array}{ccc}\begin{array}{c}\text { Alliage } \\ \mathrm{n}^{\mathrm{0}}\end{array} & \text { Etat } & \sigma_{\mathrm{R}-100 \mathrm{~h}}^{1200 \circ \mathrm{C}} \\ \overline{1} & \text { Trempé } & 1 \overline{8,0} \\ 3 & \text { Revenu } & 15,0 \\ & \text { Trempé } & 14,5 \\ & \text { Revenu } & 12,0\end{array}$

- le traitement thermo-mécanique $2 \mathrm{~h} / 2000^{\circ} \mathrm{C} /$ $\mathrm{RF}+$ écrouissage à froid de $50 \%+2 \mathrm{~h} / 2000^{\circ} \mathrm{C} /$ TH n'améliore pas sensiblement la résistance au fluage. D'autre part cette façon de faire soulève des difficultés de réalisation, l'écrouissage à froid étant difficile après le premier traitement;

- le taux d'écrouissage à froid avant le traitement final a aussi son importance: sur l'alliage $\mathrm{n}^{\mathrm{o}} 3$, l'écrouissage optimal est de $90 \%$; il est de 60 à $80 \%$ sur les alliages $n^{\text {os }} 1,2$ et 4 .

4.3 ESSAIS DE PLIAGE - Sur les alliages $n^{\circ} 1$ et 3 , nous avons déterminé le rayon de pliage minimal à $90^{\circ}$, à l'état trempé et à l'état recristallisé, sur de la tôle de $1 \mathrm{~mm}$ d'épaisseur (tableau X).

L'aptitude au pliage des deux alliages recristallisés est excellente. Elle est encore très bonne sur l'alliage $\mathrm{n}^{0} 3$ trempé. Seul l'alliage $\mathrm{n}^{0} 1$ à l'état trempé se

\section{TABLEAU X}

Rayon de pliage à $90^{\circ}$, sans fissuration

$\begin{array}{ccc}\text { Alliage } & \text { Etat trempé } & \text { Etat } \\ \overline{1} & \overline{30} e & <\bar{e} \boldsymbol{e}, 3 \\ 3 & <2 e & <0,3 e\end{array}$

plie difficilement. Si les conditions d'utilisation nécessitaient une bonne aptitude au pliage à l'ambiante, il faudrait adopter pour l'alliage $n^{0} 1$ un traitement intermédiaire.

4.4 OXYDATION EN AIR CALME. - La résistance à l'oxydation des quatre alliages a été mesurée à l'aide de la thermobalance Adamel en air calme. Les gains de poids, en $\mathrm{m} \mathrm{cm}^{-2} \mathrm{~h}^{-1}$, après $2 \mathrm{~h}$ d'exposition, sont indiqués dans le tableau XI. Les vitesses d'oxydation des alliages $n^{\text {os }} 1,2$ et 4 sont de 3 à 6 fois plus faibles que celle du niobium non allié. En revanche, l'alliage $\mathrm{n}^{0} 3$ présente deux anomalies: vitesses d'oxydation élevées à $1000^{\circ} \mathrm{C}$ et $1200^{\circ} \mathrm{C}$, et une vitesse plus faible à $1200^{\circ} \mathrm{C}$ qu’à $1000^{\circ} \mathrm{C}$.

\section{TABLEAU XI}

Vitesse d'oxydation des alliages

(exprimée en $\mathrm{mg} \mathrm{cm}^{-2} \mathrm{~h}^{-1}$, essai de $2 \mathrm{~h}$ en air calme)

$\begin{array}{ccc}\begin{array}{c}\text { Alliage } \\ \mathrm{n}^{\mathrm{O}}\end{array} & 1000^{\circ} \mathrm{C} & 1200^{\circ} \mathrm{C} \\ \frac{1}{1} & 10,5 & 27,1 \\ 2 & 13,4 & 20,9 \\ 3 & 74,0 & 41,7 \\ 4 & 7,4 & 29,7\end{array}$

4.5 CARACtÉRISTIQUeS DE TRACTION. - Les quatre alliages, à l'état trempé, ont été tractionnés à $20^{\circ} \mathrm{C}$, $780^{\circ} \mathrm{C}, 1080^{\circ} \mathrm{C}, 1200^{\circ} \mathrm{C}$ et $1300^{\circ} \mathrm{C}$. Les éprouvettes utilisées ont été prélevées dans les barres rétreintes. La vitesse de traction adoptée pour tous les essais a été de $6,6 \% / \mathrm{mn}$.

La figure 4 montre l'évolution de la charge et de

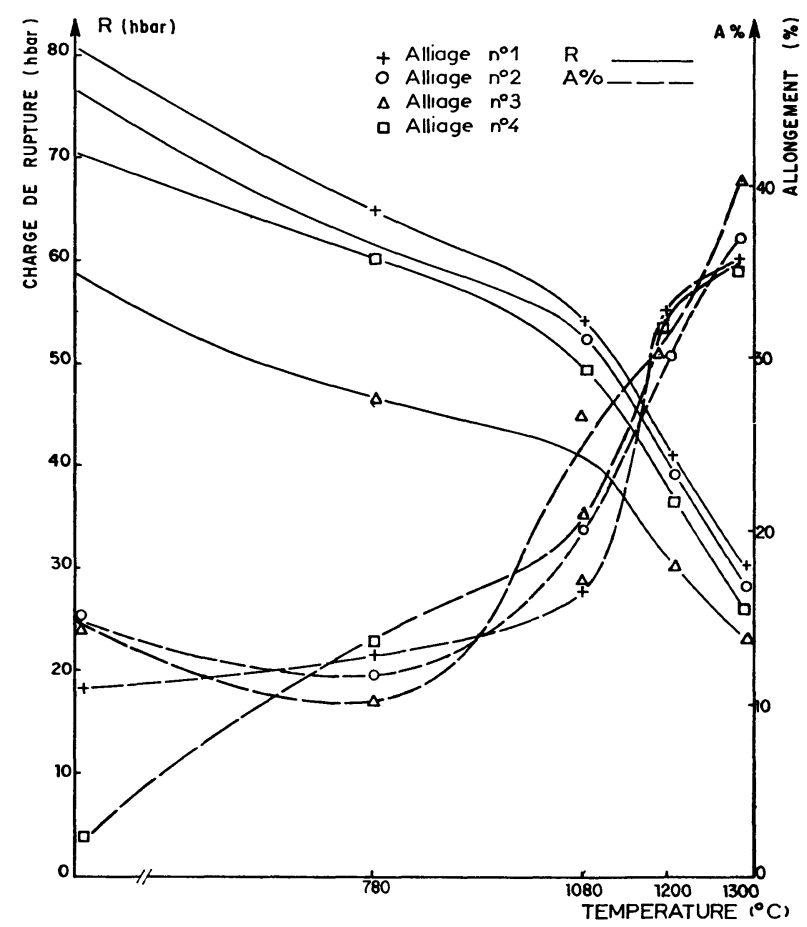

Fig. 4. - Influence de la température sur la charge et l'allongement de rupture. Barres rétreintes traitées $2 \mathrm{~h}$ à $2000^{\circ} \mathrm{C} /$ Trempe à l'huile.

l'allongement à la rupture en fonction de la température. La charge de rupture des quatre alliages baisse peu jusque vers $1080^{\circ} \mathrm{C}$; au-delà, elle décroît un peu 
plus vite. L'alliage $\mathrm{n}^{\circ} 1$ est le plus résistant avec une charge de rupture de $54 \mathrm{hbar}$ à $1080^{\circ} \mathrm{C}$ et de $40 \mathrm{hbar}$ à $1200^{\circ} \mathrm{C}$. L'alliage $\mathrm{n}^{\circ} 3$, le moins résistant, dépasse quand même 30 hbar à $1200^{\circ} \mathrm{C}$.

L'allongement de rupture sur les alliages nos 1,2 et 3 est toujours supérieur à $10 \%$. Seul l'alliage no 4 est très peu ductile à l'ambiante, du moins dans ' l'état trempé. Pour utiliser cet alliage industriellement, il faudra adopter un traitement intermédiaire entre l'état recristallisé et l'état trempé.

Sur les quatre alliages, nous avons fait des essais de traction à l'ambiante après recuit de recristallisation. Leurs caractéristiques, comparées à celles obtenues sur l'état trempé, sont indiquées dans le Tableau XII.

\section{TABLEAU XII}

Influence du traitement sur les caractéristiques de traction à l'ambiante

\begin{tabular}{|c|c|c|c|c|c|c|}
\hline \multirow{2}{*}{ 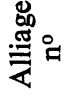 } & \multicolumn{3}{|c|}{ Etat trempé } & \multicolumn{3}{|c|}{ Etat recristallisé } \\
\hline & $R_{\text {hbar }}$ & $A \%$ & $\Sigma \%$ & $R_{\mathrm{hbar}}$ & $A \%$ & $\Sigma \%$ \\
\hline 1 & $\overline{83,3}$ & $\overline{11,9}$ & $\overline{18}$ & 80,0 & $\overline{16}$ & 40 \\
\hline 2 & 82,0 & 12,4 & 25 & 74,8 & 16,6 & 33 \\
\hline 3 & 59,3 & 11,4 & 30 & 46,7 & 14,7 & 52 \\
\hline 4 & 70,0 & 3,6 & 6 & 66,8 & 13,4 & 49 \\
\hline
\end{tabular}

4.6 CARACTÉRISTiques DE fluage. - Nous avons déterminé, sur tôles de $1 \mathrm{~mm}$ d'épaisseur et sur barres de $10 \mathrm{~mm}$ de diamètre, les contraintes donnant, respectivement, une durée de vie de $100 \mathrm{~h}$ à $1200^{\circ} \mathrm{C}$ et un allongement de fluage de $1 \%$ en $150 \mathrm{~h}$ à $1080^{\circ} \mathrm{C}$ (tableau XIII).

\section{TABLEAU XIII}

Caractéristiques de fluage, état trempé

Tôle (épaisseur : $1 \mathrm{~mm}$ )

$\begin{array}{cc}\begin{array}{c}\text { Alliage } \\ \mathrm{n}^{\mathrm{O}}\end{array} & \begin{array}{c}\sigma_{\mathrm{R}-100 \mathrm{~h}}^{1200 \mathrm{C}} \\ -\end{array} \\ \overline{1} & 18,0 \\ 2 & 14,7 \\ 3 & 14,5 \\ 4 & 17,3\end{array}$

Barre (diamètre : $10 \mathrm{~mm}$ )
Le meilleur alliage est encore l'alliage $\mathrm{n}^{0} 1$, avec un $\sigma_{\mathrm{R}-100 \mathrm{~h}}^{1} 200 \mathrm{oC}$ de 19,6 hbar et un $\sigma_{1 \%-150 \mathrm{~h}}^{1}$ de $20,2 \mathrm{hbar}$. L'alliage $\mathrm{n}^{0} 3$ a des caractéristiques qui, bien que modestes, n'en sont pas moins intéressantes eu égard à sa malléabilité.

L'ensemble des essais de fluage-rupture à $1200{ }^{\circ} \mathrm{C}$, sur tôles et sur barres montre qu'à cette température la relation liant le logarithme de la durée de vie au logarithme de la contrainte est linéaire pour les quatre alliages. Les équations de ces droites sont reportées dans le tableau XIV. Notons enfin qu'à $1200^{\circ} \mathrm{C}$, le temps de rupture est relié à la vitesse minimale de fluage par une relation simple (Fig. 5).

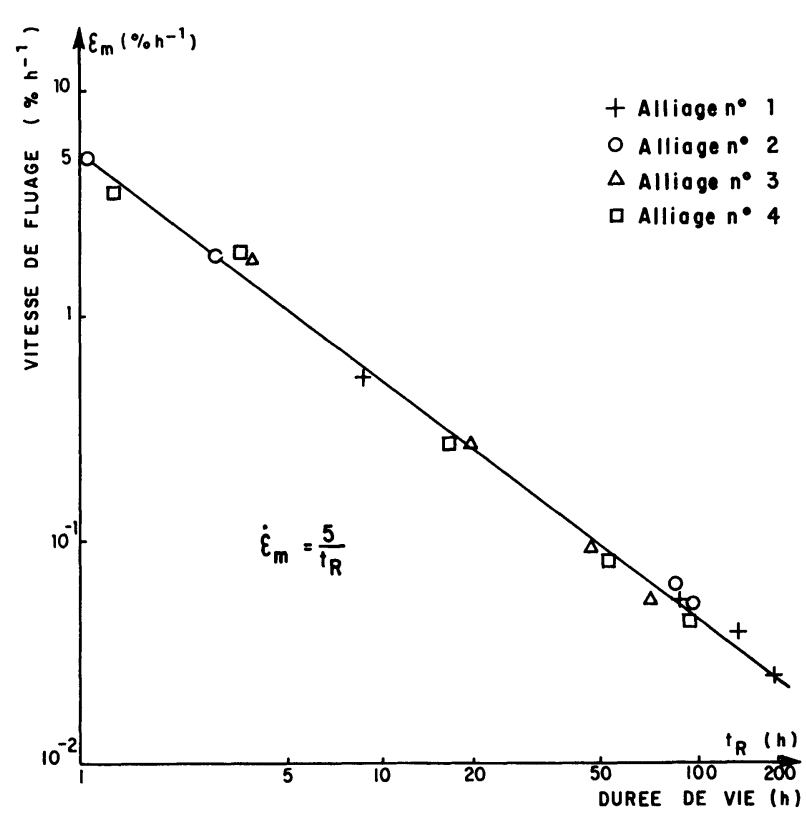

FIG. 5. - Relation $\left(t_{\mathrm{R}}, \dot{\varepsilon \mathrm{m}}\right)$.

4.7 Mesures De DENSITÉ. - Elles ont été faites par pesée dans l'air et dans l'eau. C'est l'alliage $n^{0} 1$ qui a la plus forte densité (tableau $X V$ ) suivi de l'alliage $\mathrm{n}^{\mathrm{o}} 2$.

Les alliages $\mathrm{n}^{\circ} 3$ et 4 ont des densités voisines de celles du niobium non allié.

\section{TABLEAU XIV}

Relation $\left(\sigma, t_{\mathrm{R}}\right) \grave{a} 1200^{\circ} \mathrm{C}$

$\begin{gathered}\text { Alliage } \\ \mathrm{n}^{\mathrm{o}}\end{gathered}$
-
1
2
3
4

Tôle

$$
\begin{aligned}
& \text { (épaisseur : } 1 \mathrm{~mm} \text { ) } \\
& \text { - } \\
& \log \sigma=-0,1268 \log t_{\mathrm{R}}+1,4793 \\
& \log \sigma=-0,1365 \log t_{\mathrm{R}}+1,4413 \\
& \log \sigma=-0,0991 \log t_{\mathrm{R}}+1,3587 \\
& \log \sigma=-0,0923 \log t_{\mathrm{R}}+1,4216
\end{aligned}
$$

Barre

(diamètre : $10 \mathrm{~mm}$ ) 
TABLEAU XV

Densité des alliages $n^{\text {os }} 1$ à 4

$\begin{array}{cc}\begin{array}{c}\text { Alliage } \\ \mathrm{n}^{0}\end{array} & \text { Densité } \\ \overline{1} & \overline{-} \\ 2 & 9,30 \\ 3 & 9,02 \\ 4 & 8,72 \\ & 8,57\end{array}$

4.8 EXAMENS MICROGRAPHIQUES. - L'étude structurale de l'alliage $\mathrm{n}^{\mathrm{o}} 1$ nous a permis de mieux comprendre le comportement de cet alliage et l'évolution de ses caractéristiques en fonction du traitement thermique.

L'alliage coulé et homogénéisé $8 \mathrm{~h}$ à $1450^{\circ} \mathrm{C}$ a une structure de fonderie grossière (Fig. 6) où de gros carbures parsèment la matrice. Cette matrice est une solution solide $\mathrm{Nb}-\mathrm{W}-\mathrm{Mo}$.

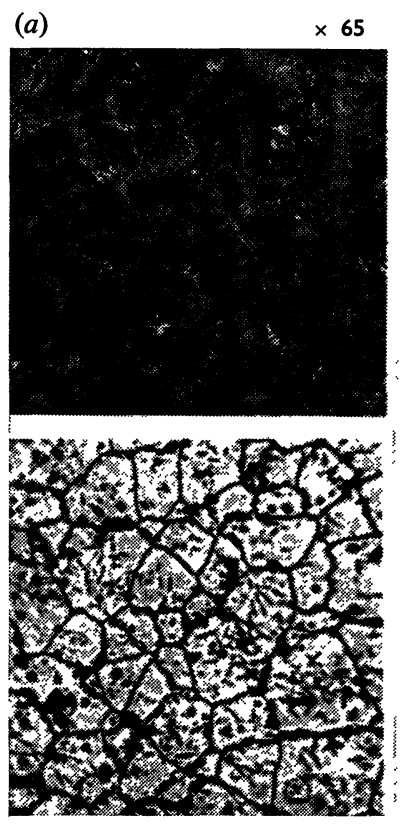

(c)

$\times 800$
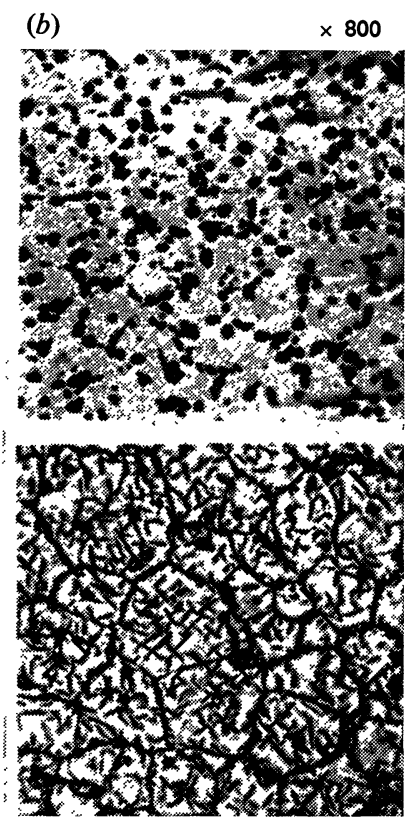

(d)

$\times 800$

FIG. 6. - Structure micrographique de l'alliage $\mathrm{n}^{\circ} 1$ :

a) Coulée, Homogénéisé $8 \mathrm{~h}$ à $1450^{\circ} \mathrm{C}$.

b) Laminé. $1 \mathrm{~mm}$ d'épaisseur. Traité $2 \mathrm{~h}$ à $2000^{\circ} \mathrm{C}$. Refroidissement lent.

c) Laminé. $1 \mathrm{~mm}$ d'épaisseur. Traité $2 \mathrm{~h}$ à $2000^{\circ} \mathrm{C}$. Trempe huile.

d) Laminé. $1 \mathrm{~mm}$ d'épaisseur. Traité $2 \mathrm{~h}$ à $2000^{\circ} \mathrm{C}$. Trempe huile $+4 \mathrm{~h}$ à $1250^{\circ} \mathrm{C}$.

Le corroyage affine la structure de fonderie, sans modifier la taille des carbures. Ceux-ci, sur les tôles laminées, ne se remettent en solution qu'au cours du maintien à $2000^{\circ} \mathrm{C}$ (sauf les plus gros). La structure finale dépend alors du mode de refroidissement : si celui-ci est lent, la précipitation des carbures est grossière ; au contraire, un refroidissement rapide provoque une précipitation fine, d'autant plus fine qu'il est plus rapide.
Les phases précipitées sont de deux types : une phase $\mathrm{NbC}$ cubique à faces centrées de paramètre $a=4,46 \AA$ qui correspond aux gros carbures de l'état coulé et d'une façon générale aux carbures formés lentement par globulisation; une phase $(\mathrm{Nb}, \mathrm{Hf}) \mathrm{C}$, de paramètre $a=4,55 \AA$ très fine après la trempe à l'huile, mais qui grossit au cours du revenu de $4 \mathrm{~h}$ à $1400^{\circ} \mathrm{C}$. Elle apparaît alors nettement en diffraction X (Fig. 7).

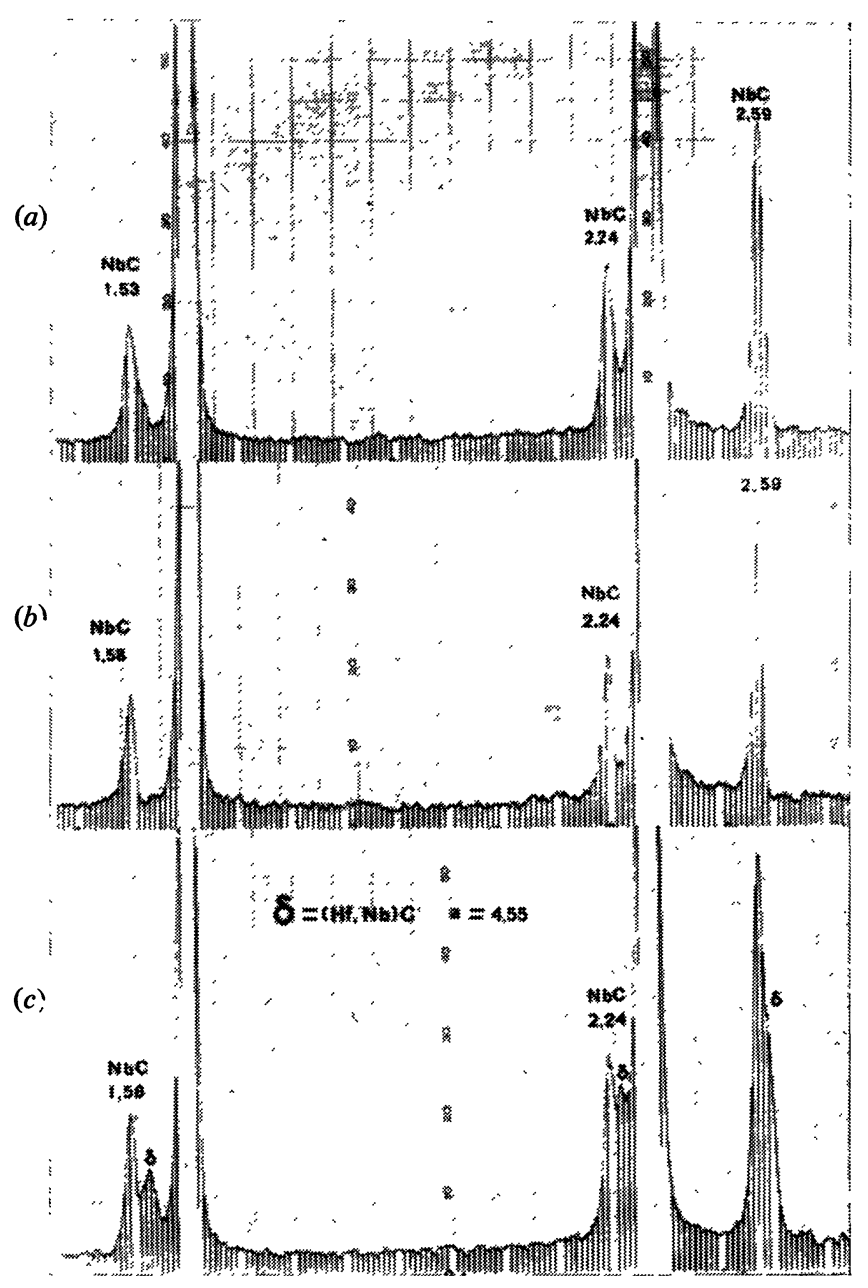

FIG. 7. - Identification des phases dispersées dans l'alliage $\mathrm{n}^{\mathrm{o}} 1$. Examen au diffractomètre à compteur. Radiation utilisée : $\mathrm{CuK}_{\alpha}$.

a) Etat $2 \mathrm{~h}$ à $2000^{\circ} \mathrm{C}$. Refroidissement lent.

b) Etat $2 \mathrm{~h}$ à $2000^{\circ} \mathrm{C}$. Trempe huile.

c) Etat $2 \mathrm{~h}$ à $2000^{\circ} \mathrm{C}$. Trempe huile $+4 \mathrm{~h}$ à $1400^{\circ} \mathrm{C}$.

Ainsi, l'évolution des caractéristiques mécaniques en fonction du traitement thermique s'explique convenablement : le traitement à $2000^{\circ} \mathrm{C}$ suivi d'un refroidissement lent, par la globulisation qu'il provoque et l'épuisement de la matrice en carbone, améliore considérablement la malléabilité. Au contraire, le refroidissement rapide donne une précipitation fine et uniforme qui bloque efficacement les dislocations et augmente la résistance au fluage. Plus précisément, la précipitation après la trempe à l'huile est sans doute incomplète. Il se produit alors, au cours du fluage à $1200^{\circ} \mathrm{C}$, un revenu sous contrainte qui achève de précipiter la fraction restée en solution. Enfin, le 
survieillissement est déjà sensible après un revenu de $4 \mathrm{~h}$ à $1400^{\circ} \mathrm{C}$.

5. Conclusions. - Cette recherche nous a permis de faire de réels progrès dans le développement des alliages de niobium résistant à chaud. Ces progrès portent sur plusieurs points :

- La composition. - L'association du molybdène et du tungstène dans le durcissement de solution solide permet de durcir davantage la matrice en conservant une densité raisonnable. L'utilisation des phases $\mathrm{HfC}$ et $\mathrm{HfB}_{2}$ s'est révélée très efficace, davantage même que la phase $\mathrm{ZrC}$ couramment employée. L'abandon des éléments à faible tension de vapeur ( $\mathrm{Ti}, \mathrm{V}, \mathrm{Al})$ a amélioré la santé des lingots et facilité le contrôle des compositions.

- L'analyse. - L'analyse chimique classique, mal adaptée au dosage du $\mathrm{Ti}$, du V, du $\mathrm{Zr}$, de l'Hf, du W et du Mo dans ces alliages, a souvent conduit par le passé à des imprécisions dans la détermination des teneurs, ce qui a embrouillé parfois l'appréciation de l'influence des éléments d'addition.

La mise au point d'une analyse par fluorescence $X$, en permettant le contrôle rapide et précis des compositions, a donc été déterminante dans cette recherche.

- Les traitements thermiques. - L'importance du refroidissement après traitement a été clairement établie. Un traitement de $2 \mathrm{~h}$ à $2000^{\circ} \mathrm{C}$ suivi d'un refroidissement lent améliore la malléabilité en cours de transformation, aloıs que le même traitement suivi d'une trempe à l'huile augmente les caractéristiques de fluage du produit fini.

Quatre alliages ont été mis au point :

- le WD 15 (9 W 6 Mo 4 Hf 0,2 C) (alliage ${ }^{\circ} 1$ ),

- le WD 13 (11 W 2 Mo $2 \mathrm{Zr} 2 \mathrm{Hf} \quad 0,3 \mathrm{C})$ (alliage $n^{0} 2$ ),

$$
\text { - le W } 6 \text { B (6 W } 2 \text { Zr } 2 \text { Hf 0,2 C 0,2 B) (alliage }
$$
no 3),

$$
\text { - le D } 9 \text { (9 Mo } 2 \text { Zr 0,2 C (alliage no 4). }
$$

Ces alliages sont réellement transformables, à condition de respecter les processus de transformation définis au cours de cette étude.

A l'état trempé, leurs caractéristiques de fluage sont excellentes et leur ductilité reste acceptable, même à l'ambiante, sauf pour l'alliage D $9(6 \%$ de striction à l'ambiante à l'état traité). Pour cet alliage, il faudrait adopter un traitement à plus basse température.

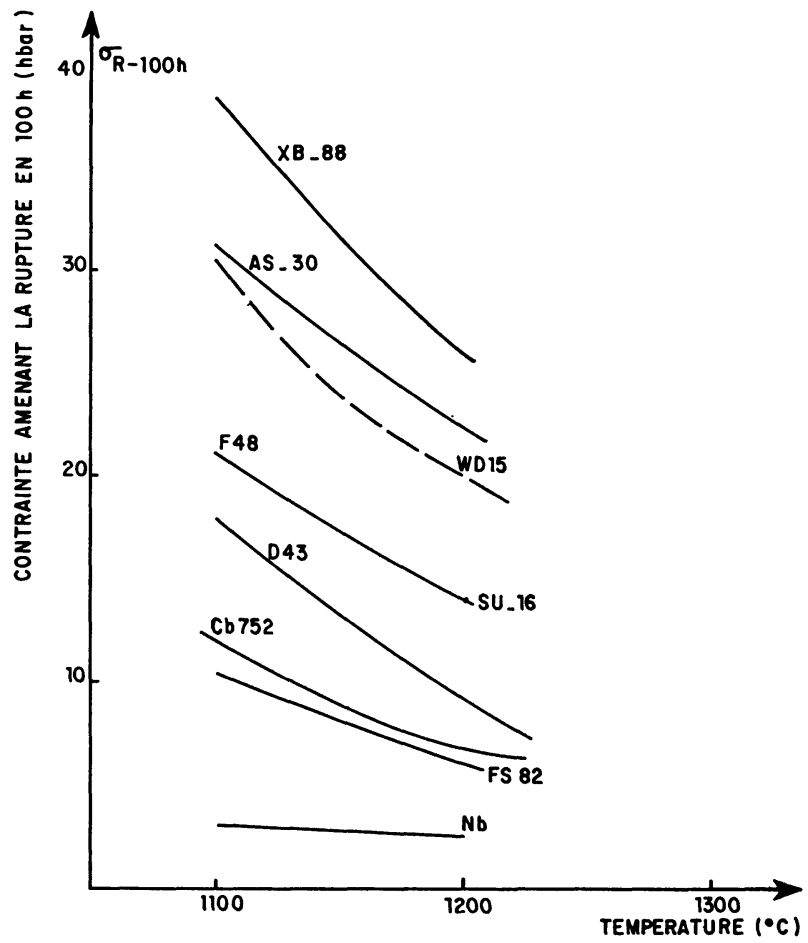

FIG. 8. - Comparaison du WD 15 aux autres alliages de niobium existants.

Industriellement, la trempe à l'huile peut présenter des difficultés de réalisation; mais comme on l'a souligné, le traitement au four avec coupure brutale du chauffage procure sensiblement la même résistance au fluage. Enfin, le développement industriel de l'alliage W 6 B nécessiterait une étude complémentaire pour résoudre les difficultés d'élaboration et d'analyse rencontrées.

Ces réserves faites, les quatre alliages retenus présentent des compromis intéressants de malléabilité et de résistance au fluage. L'alliage W D 15, notamment, a une tenue à chaud qui dépasse la plupart des alliages actuels : sur la figure 8 , où sont portées les valeurs de $\sigma_{\mathrm{R}-100 \mathrm{~h}}$ en fonction de la température, on constate que le W D 15 vient aussitôt après l'AS 30 et le X B 88 qui ont, en revanche, des densités plus élevées.

Remerciements. - Cette étude a été réalisée grâce à un contrat passé avec la D. G. R. S. T. (marché 67.00.495). Nous remercions cet organisme de nous avoir aidé financièrement dans cette recherche.

\section{Bibliographie}

Briggs (D. C.), A Survey of Niobium Alloys and their Strengthening Mechanisms, Department of Mines and Technical Surveys, Ottawa, IC 153, 1963.

Mc Quillan et Clark (P. V. M.), The Relation between Structure and Mechanical Properties in the Development of New High Temperature Alloys, National Physical Laboratory Symposium no 15, 1963.

SYRe (R.), Métaux réfractaires et leurs alliages, Agardograph, 1964.

BeGLel et BECHTOLD, Effect of Alloying on the Mechanical Properties of Niobium, J. Less Common Metals, $1961,3,1$.
Bartlett (E. S.), High Temperature Solid-SolutionStrengthened Colombium Alloy, Trans. AIME, $1963,227,459$.

Delgrosso (E. J.) et al., Development of Niobium-Zirconium-Carbon Alloys, J. Less Common Metals, 1967, 12, 173.

Mc AdAm (G. D.), The Influence of Carbide and Boride Additions on the Creep Strength of Niobium Alloys, J. Inst. Metals, 1968, 96, 13.

RANSON (J. D. W.) and ARGEN (B. B.), The Effect of Oxygen and Carbon on the Creep Strength of Niobium, J. Inst. Metals, 1967, 95, 212. 